\title{
MANAGEMENT OF POST CABG STERNAL OESTEOMYELITIS
}

\author{
A.K. Deb ${ }^{a}$, M.U. Akand ${ }^{b}$, M.A. Islam ${ }^{c}$
}

\begin{abstract}
:
Two cases of post CABG sternal wound with sternal osteomyelitis were managed in plastic surgery unit from year 2007-2008. One of them was male and other was female. Both were diabetic. The male one had multiple sinus tracts with discharge and the female one had well granulated wound with discharge. Both the cases were managed by adequate debridement of the wound with removal of some loose sternal wire and placement of a cutaneous flap over the wound. In both cases, the flaps survived well, infection was completely resolved. The scar over the chest wall was cosmetically acceptable.
\end{abstract}

\section{INTRODUCTION:}

Sternal wound infection with osteomyelitis is an infrequent, yet potentially devastating complication following coronary artery bypass grafting $(\mathrm{CABG})$. This complication is associated with amplified cost of care, prolonged hospitalization, and significant morbidity, with reported mortality rates of more than of $50 \%{ }^{1}$ Sternal dehiscence in early days was treated conservatively with open drainage and debridement. Later on, closed management with negative pressure dressing and continuous antibiotic irrigation was introduced; however, the mortality rate remained approximately $20 \%{ }^{1}$ The use of vascularized regional tissue for coverage allows greater blood flow, obliteration of dead space, and faster healing time because of quicker resolution of infection. Today, the management of sternal dehiscence and infection involves wide

a. Brig. Gen. Anjan Kumar Deb (Retd), MBBS, FCPS (SURGERY), Fellowship Plastic surgery, Singapore, Ex-Adviser Specialist in Burn \& Reconstructive surgery, CMH Dhaka Cantonment, Dhaka. Honorary Consultant, Plastic \& Reconstructive Surgery, BIRDEM Hospital, Dhaka.

b. Dr.Morshed Uddin Akand, MBBS, Senior Medical Officer, Plastic \& Reconstructive Surgery, BIRDEM Hospital, Dhaka.

c. Dr. Md. Anwarul Islam, MBBS, Medical Officer, Plastic \& Reconstructive Surgery, BIRDEM Hospital, Dhaka, Department of Plastic \& Reconstructive Surgery, BIRDEM Hospital Dhaka.

Address of correspondence: Dr.Brig.Gen Anjan Kumar Deb (retd), Plastic Surgery Dept. Room No: 1119, BIRDEM Hospital, Shahabag, Dhaka-1000. E-mail:akd483@gmail.com

Cell phone:01819211394. debridement of devitalized infected soft tissue and bone, culture-specific antibiotics, and flap closure to achieve wound healing. Thus, the mortality rate from sternal wound dehiscence dropped to less than $8-10 \%{ }^{1}$ We have successfully treated 2 patients who developed deep sternal wound infection after cardiac surgery by combining onestage debridement with cutaneous transposition flap coverage of the wound and continuous irrigation with antibiotic solution.

\section{CASE -1}

A 65 years old female diabetic patient with sternal wound (Fig-1) was admitted to Depterment of Plastic Reconstructive Surgery, BIRDEM on 31st December 2007. She had undergone coronary bypass surgery on 15th July 2007 at a Private Hospital. After one month she developed wound infection followed by dehiscence and was re-admitted in the same hospital. There she was treated by daily dressing for one month and then secondary closure was done, but she again developed wound dehiscence because of infection and sternal osteomyelitis. On admision the wound

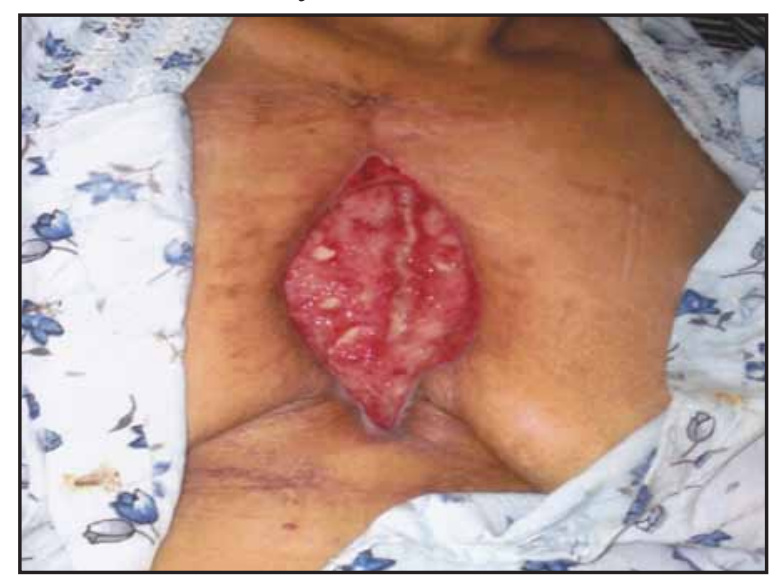

Fig -1: Case 1, wound at presentation. 
was well granulated with exposure of sternum and margin was inverted. There was whitish discharge, containing granular bony particles, and whitish slough on the floor of the wound (Fig-2). Her routine investigations were within

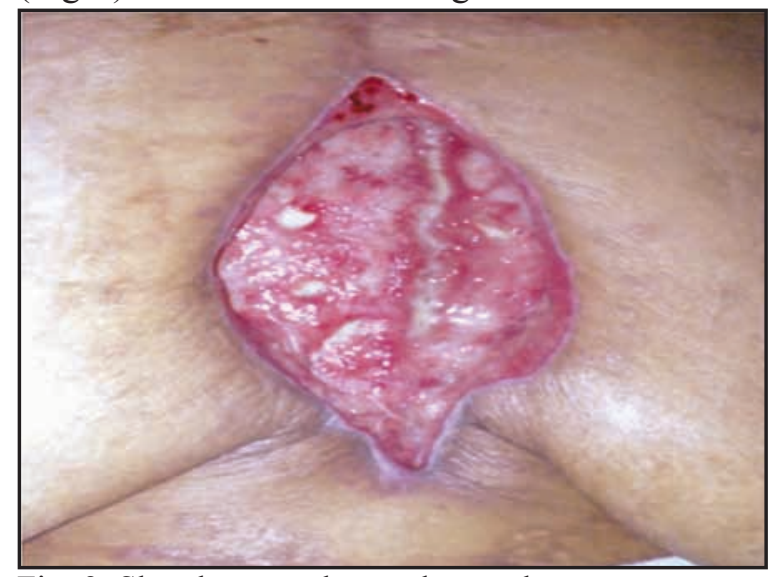

Fig -2: Slough covered sternal wound.

normal limit, echocardiogram showed a LVEF of $65 \%$. Wound swab for culture and sensitivity, showed presence of staphylococcus aureus and pseudomonus $\mathrm{sp}$, sensitive to imipenum.

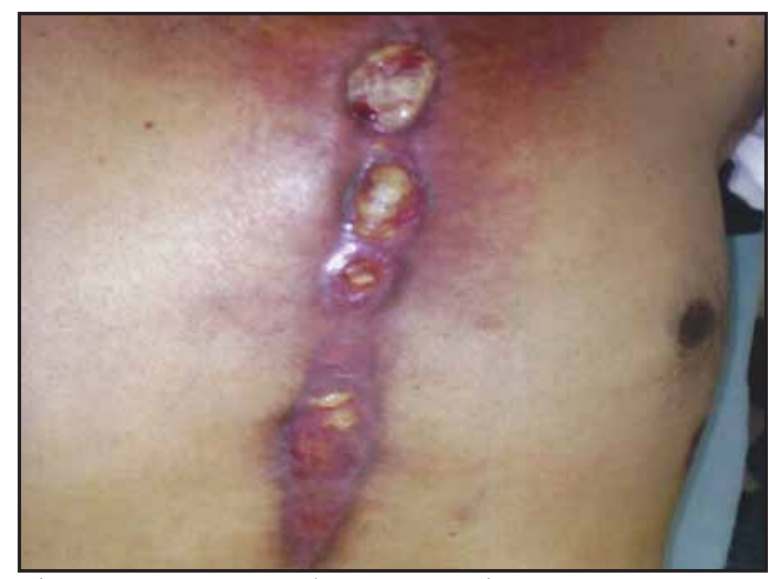

Fig -3: Case 2, wound at presentation.

\section{CASE-2}

A 57 years old male diabetic patient with multiple discharging sinuses over the sternum, (Fig-3 ) was admitted on 7th August 2008 in our unit at BIRDEM. He had undergone coronary bypass surgery on 10th June 2008 in a Government Institute for Coronary and Vascular Disease. During the operation he had a cerebral infarction and left sided hemiplegia. One month later he developed wound infection with multiple discharging sinuses. He then visited to the same hospital and was admitted there, daily dressing was done but the wound was not improving. His diabetic state was poorly controlled. On examination in our hospital we found multiple discharging sinuses over the chest wall with spouting growth of granulation tissue. There was whitish discharge from the sinuses. All the routine investigations were within normal limit. Echocardiogram, showed a LVEF of $50 \%$ but his diabetes was uncontrolled. Pus for culture and sensitivity showed presence of staphylococcus aureus , sensitive to Ciprofloxacin, Amikacin, Gentamycin, Imipenem.

\section{OPERATIVE PROCEDURE}

\section{Anaesthesia:}

General anaesthesia with ETT, full muscle relaxation, closed circuit mechanical ventilation was used.

\section{Position: Supine}

\section{Flap Design \& Composition:}

A rhomboid flap was marked on the lower inner quadrant of the right breast. The sides of which were half of the sides of the defect and the short diagonal equal to the maximum width of the defect after debridement. The flap was raised deep to the superficial fascia avoiding the breast tissue (Fig-4).

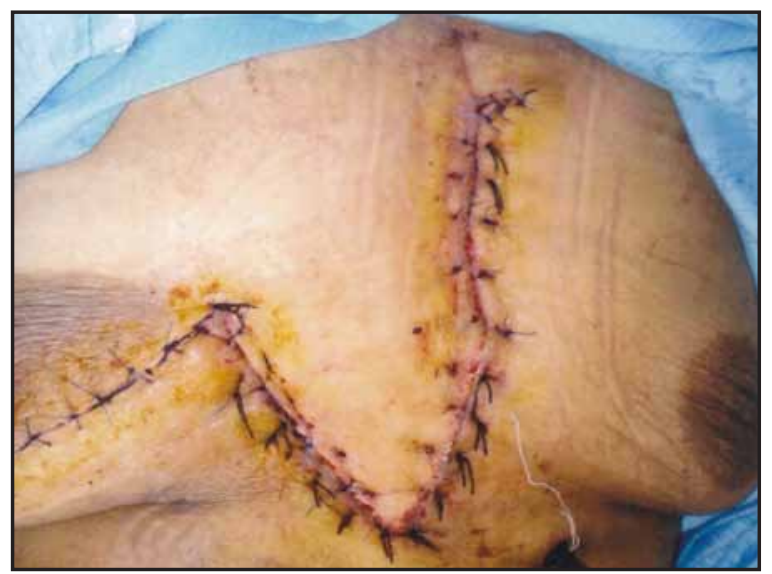

Fig -4: Case 1, after flap coverage. 


\section{Flap Inset and Donor site Repair:}

The wound was debrided by scraping off the granulation tissue, freshening of the margins and removal of all the loosened wires. The flap was then sutured to the margin of the wound keeping an irrigating and drainage tube underneath the flap. The donor defect could be closed directly by undermining $\&$ advancement.

A drain underneath the flap in case 1 and an irrigation drain in the case 2 were placed. Gentamycin $80 \mathrm{mg}$ in $500 \mathrm{ml}$ normal saline was irrigated through irrigation tube @15 drops per minute for 5 days (Fig.-5).

\section{Post Operative Period:}

Post operative period was uneventful in both the cases. We removed the irrigation and drainage tube on 14th POD. All stitches were removed on 21 st POD and the patient was discharged with oral levofloxacin for 6 weeks and other medications.

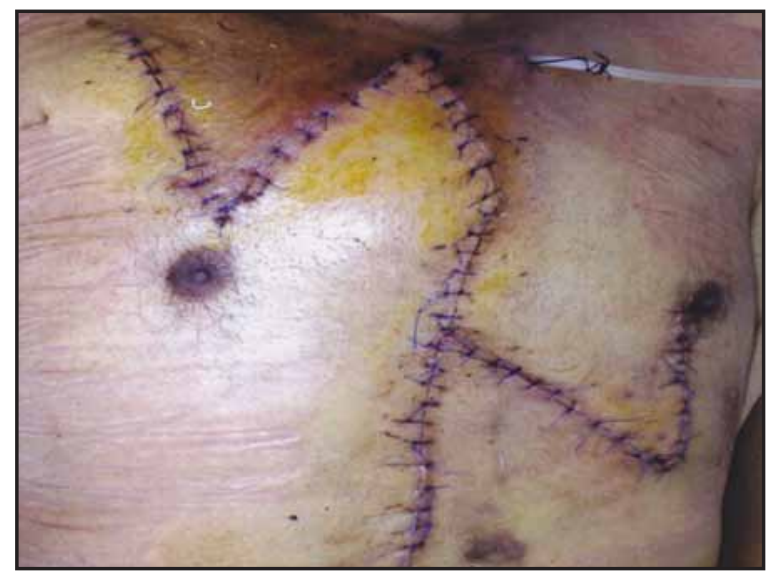

Fig -5: Immediate post operative pitcurer of case 2.

\section{Follow up:}

The patients were followed up monthly for 6 months and then after one year and now they are doing well (Fig.-6 \& 7).

\section{DISCUSSION:}

Deep sternal wound infection after cardiac surgery continues to represent an important complication associated with high morbidity and cost. A multicenter prospective trial has established that the independent risk factors

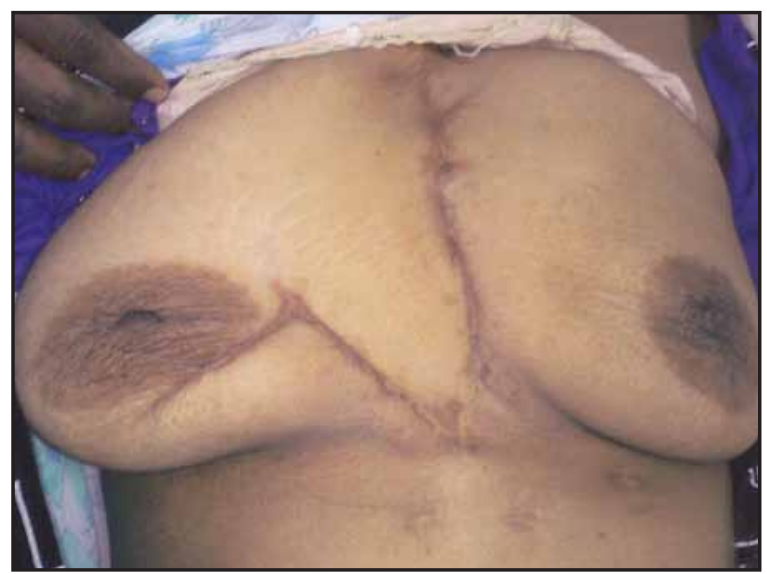

Fig -6: Post operative outcome of case 1.

for sternal wound infection include obesity, $\mathrm{CABG}$, re-operation, and postoperative inotropic support. ${ }^{2}$ Other postoperative variables such as nephrological and pulmonary complications have also been found to be strong predictors of sternal

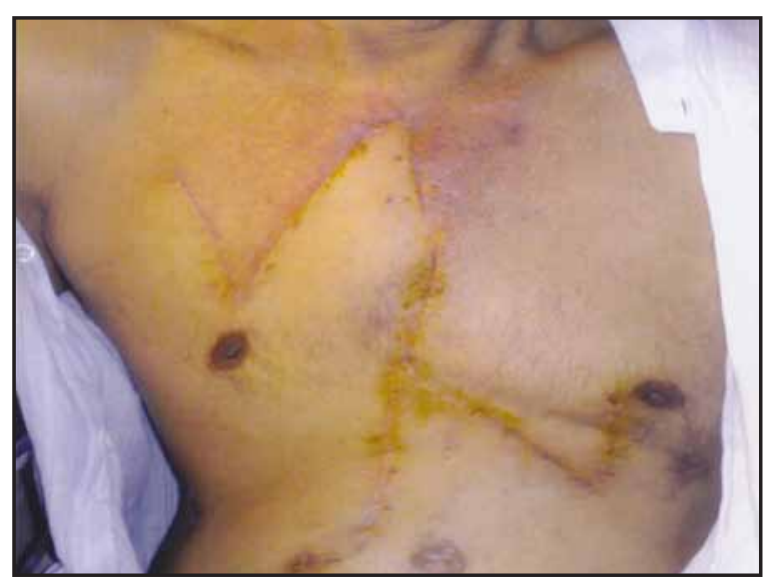

Fig -7: Post operative outcome of case 2.

wound infection. ${ }^{3}$ The risk factors for mortality in patients who develop deep sternal wound infection have been determined as bacteremia, intra-aortic balloon pumping, advanced age, and prolonged mechanical ventilation. ${ }^{4}$ However, the majority of the deaths are secondary to sepsis and multi-organ failure.

During the development of modern cardiac surgery, a number of wound-healing strategies have been established for the treatment of poststernotomy mediastinitis. Conventional forms of treatment usually involve surgical revision with open dressings or closed irrigation, 
or reconstruction with vascularized soft tissue flaps such as omentum or pectoral muscle or vacuum-assisted closure. These wound-healing techniques may be used as a single-line therapy or in combination with other procedures. At the present time, there is little consensus regarding the appropriate surgical approach to mediastinitis following open-heart surgery.

Vacuum-assisted closure (VAC) is a recent technical innovation in wound care with a growing number of applications. This wound-healing system was developed in the U.S. by Argenta and Morykwas in the mid 1990s. This wound-healing technique is based on the application of local negative pressure to a wound. This is achieved by placing polyurethane foam with an open pore structure of $400-600 \mu \mathrm{m}$ in the wound. One end of a non-collapsible tube is then connected to the foam and the other end is connected to a vacuumsource in a closed system connected to a fluid container. The exact mechanism by which VAC exerts its wound-healing mechanism is not fully understood. And this therapy is not suitable for wound loaded with high count of bacteria.

An important advance in mediastinitis treatment was made in 1963 with continuous irrigation in combination with drainage and a closed sternum. ${ }^{5}$ Bryant et al. achieved further development with antibiotic irrigation. ${ }^{6}$ Surgical revision with rewiring or closed irrigation offers an expeditious procedure with the advantage of a closed wound and a stable sternum. We used antibiotic irrigation in one case and got good result.

The optimum treatment of sternal infection has been the subject of considerable debate. Sternal osteomyelitis can be managed by simple cutaneous flap transposition with appropriate post operative long term antibiotic therapy. Simpler techniques may give better prognosis and less post operative morbidity and complication which may happen in muscle flap or in omental flap. Studies with large number of cases may give a definitive conclusion.

\section{Reference :}

1. Chest Reconstruction, Sternal Dehiscence Author: Sanjay K Sharma, MD, Consulting Staff, Department of Plastic Surgery, Meridian Plastic Surgery Center, Seton Medical Center Contributor Information and Disclosures (e-medicine, medscape) Updated: May 4, 2006.

2. The Parisian Mediastinitis Study Group. Risk factors for deep sternal wound infection after sternotomy: a prospective, multicenter study. J Thorac Cardiovasc Surg 1996;111:1200-7.

3. Noyez L, van Druten JA, Mulder J, Schroen AM, Skotnicki SH, Brouwer RM. Sternal wound complications after primary isolated myocardial revascularization: the importance of the post-operative variables. Eur $\mathrm{J}$ Cardiothorac Surg 2001;19:471-6.

4. Milano CA, Kesler K, Archibald N, et al. Sexton DJ, Jones RH. Mediastinitis after coronary artery bypass graft surgery. Risk factors and long-term survival. Circulation 1995;92:2245-51.

5. Shumacker Jr. HB, Mandelbaum I. Continuous antibiotic irrigation in the treatment of infection. Arch Surg 1963;86:384-387.

6. Bryant LR, Spencer FC, Trinkle JK. Treatment of median sternotomy infection by mediastinal irrigation with an antibiotic solution. Ann Surg 1969;169:914-920. 